\title{
Juno, the angular momentum of Jupiter and the Lense-Thirring effect
}

\author{
Lorenzo Iorio \\ INFN-Sezione di Pisa. Permanent address for correspondence: Viale Unità di Italia 68, \\ 70125, Bari (BA), Italy. E-mail: lorenzo.iorio@libero.it
}

Received — 


\begin{abstract}
The recently approved Juno mission will orbit Jupiter for one year in a highly eccentric $\left(r_{\min }=1.06 R_{\mathrm{Jup}}, r_{\max }=39 R_{\mathrm{Jup}}\right)$ polar orbit $(i=90 \mathrm{deg})$ to accurately map, among other things, the jovian magnetic and gravitational fields. Such an orbital configuration yields an ideal situation, in principle, to attempt a measurement of the general relativistic Lense-Thirring effect through the Juno's node $\Omega$ which would be displaced by about $570 \mathrm{~m}$ over the mission's duration. Conversely, by assuming the validity of general relativity, the proposed test can be viewed as a direct, dynamical measurement of the Jupiter's angular momentum $S$ which would give important information concerning the internal structure and formation of the giant planet. The long-period orbital perturbations due to the zonal harmonic coefficients $J_{\ell}, \ell=2,3,4,6$ of the multipolar expansion of the jovian gravitational potential accounting for its departures from spherical symmetry are a major source of systematic bias. While the Lense-Thirring node rate is independent of the inclination $i$, the node zonal perturbations vanish for $i=90$. In reality, the orbit injection errors will induce departures $\delta i$ from the ideal polar geometry, so that the zonal perturbations will come into play at an unacceptably high level, in spite of the expected improvements in the low-degree zonals by Juno. A linear combination of $\Omega$, the periJove $\omega$ and the mean anomaly $\mathcal{M}$ cancels out the impact of $J_{2}$ and $J_{6}$. A two orders of magnitude improvement in the uncanceled $J_{3}$ and $J_{4}$ would be needed to reduce their bias on the relativistic signal to the percent level; it does not seem unrealistic because the expected level of improvement in such zonals is three orders of magnitude.
\end{abstract}

Subject headings: Relativity and gravitation; Lunar, planetary, and deep-space probes; Jupiter; Gravitational fields 


\section{Introduction} spinning, solar powered spacecraft to be placed in a highly eccentric polar orbit around Jupiter (see Table 1 for its relevant orbital parameters) specifically designed to avoid its highest radiation regions. Understanding the formation, evolution and structure of Jupiter is the primary science goal of Juno. It will carry onboard a dual frequency gravity/radio

Table 1: Planetocentric nominal orbital parameters of Juno. $a, e, i$ are the semi-major axis (in jovian radii $R=71492 \mathrm{~km}$ ), the eccentricity and the inclination (in deg) to the Jupiter's equator, respectively. $P$ is the orbital period (in days). $T$ is the mission duration (in years).

\begin{tabular}{ccccc}
\hline$a(R)$ & $e$ & $i(\operatorname{deg})$ & $P(\mathrm{~d})$ & $T(\mathrm{yr})$ \\
\hline 20.03 & 0.947 & 90 & 11 & 1 \\
\hline
\end{tabular}

science system, a six wavelength microwave radiometer for atmospheric sounding and composition, a dual-technique magnetometer, plasma detectors, energetic particle detectors, a radio/plasma wave experiment, and an ultraviolet imager/spectrometer. The nominal mission's lifetime is 1 year. Juno is aimed, among other things, at accurately mapping the gravitational field of Jupiter with unprecedented accuracy (Anderson et al. 2004) by exploiting the slow apsidal precession of its 11-day orbit.

In this paper we wish to explore the possibility offered by Juno to perform a test of general relativity by directly measuring the gravito-magnetic Lense-Thirring effect; its basics are reviewed below. Even putting aside the more or less successful attempts so far performed with other natural and artificial test particles orbiting different central bodies, it must be recalled that a satisfactorily empirical corroboration of a fundamental theory like

\footnotetext{
${ }^{1}$ See on the WEB http://juno.wisc.edu/index.html
} 
general relativity requires that as many independent experiments as possible are conducted by different scientists in different laboratories; thus, it is worthwhile to try to use different gravitational fields to perform such a test of intrinsic gravito-magnetism. Conversely, since, as we will see, the Lense-Thirring precessions are due to the proper angular momentum $\boldsymbol{S}$ of the orbited central body, one may also assume the existence of the general relativistic gravito-magnetism and consider such a test as a direct, dynamical measurement of the Jupiter's angular momentum through the Lense-Thirring effect; this would yield further, important information concerning the interior of Jupiter. Indeed, the moment of inertia ratio $C / M R^{2}$ entering $S$ is a measure of the concentration of mass towards the center of the planet (Irwin 2003). Such a figure, together with the measured values of the zonal2 coefficients of the gravity field accounting for its deviations from spherical symmetry may be fitted with internal models that model how the density, pressure, temperature and composition vary with depth (Irwin 2003; Guillot 2005; Hori et al. 2008). Moreover, a dynamical, model-independent determination of $S$ would be important also for a better knowledge of the history and formation of Jupiter (Machida et al. 2008).

Let us, now, briefly review the basics of the Lense-Thirring effect. In its weak-field and slow-motion approximation, the field equations of general relativity get linearized looking like those of the Maxwellian electromagnetism. Analogously with the magnetic field generated by moving electric charges, mass-energy currents give rise to a gravito-magnetic field $\boldsymbol{B}_{\mathrm{g}}$ (Mashhoon 2007); far from an isolated spinning body of mass $M$ and proper angular momentum $\boldsymbol{S}$ it is (Lichtenegger and Iorio 2007)

$$
\boldsymbol{B}_{\mathrm{g}}=-\frac{G}{c r^{3}}[\boldsymbol{S}-3(\boldsymbol{S} \cdot \hat{\boldsymbol{r}}) \hat{\boldsymbol{r}}]
$$

where $G$ is the Newtonian gravitational constant and $c$ is the speed of light in vacuum. $\boldsymbol{B}_{\mathrm{g}}$

\footnotetext{
${ }^{2}$ They preserve the axial symmetry.
} 
exerts the non-central Lorentz-like acceleration (Mashhoon 2007)

$$
\boldsymbol{A}^{\mathrm{GM}}=-\frac{2}{c} \boldsymbol{v} \times \boldsymbol{B}_{\mathrm{g}}
$$

upon a test particle moving with velocity $\boldsymbol{v}$. For ordinary astronomical bodies like, e.g., a planet, $A^{\mathrm{GM}}$ is many orders of magnitude smaller than the Newtonian monopole $A^{\mathrm{N}}=G M / r^{2}$, so that it can be considered as a small perturbation. As a result, the longitude of the ascending node $\Omega$ and the argument of pericentre $\omega$ of a test particle undergo small secular precessions (Lense and Thirring 1918)

$$
\begin{aligned}
& \dot{\Omega}_{\mathrm{LT}}=\frac{2 G S}{c^{2} a^{3}\left(1-e^{2}\right)^{3 / 2}}, \\
& \dot{\omega}_{\mathrm{LT}}=-\frac{6 G S \cos i}{c^{2} a^{3}\left(1-e^{2}\right)^{3 / 2}} .
\end{aligned}
$$

Concerning a direct measurement of the Lense-Thirring effect, attempts have been recently performed with the LAGEOS satellites in the gravitational field of the Earth (Ciufolini and Pavlis 2004; Ries et al. 2008), the Mars Global Surveyor probe orbiting Mars (Iorio 2006) and some of the inner planets of the Solar System (Iorio 2008). The evaluation of the total accuracy in some of such tests has raised a debate in the recent past (Iorio 2009a; Krogh 2007) because of the difficulty of realistically assessing the impact of certain competing dynamical effects acting as sources of systematic errors; for example, the total accuracy of the LAGEOS test may be as large as some tens percent (Iorio 2009a); the same shortcomings may affect also the approved LARES mission (Iorio 2009a, b). For an overview of several theoretical and experimental features of gravito-magnetism see, e.g., (Iorio 2007). Concerning the jovian scenario, Lense and Thirring (1918) originally proposed to use the orbital precessions of the Galilean satellites; such a possibility has been recently investigated by Iorio and Lainey (2005), but it seems to be still premature. Haas and Ross (1975) proposed a spacecraft-based experiment in the gravitational field of Jupiter to measure another gravito-magnetic effect, i.e. the precession of a gyroscope (Pugh 1959; Schiff 1960). 
It is also one of the goals of the GP-B mission (Everitt 1974) whose target was a $\approx 1 \%$ measurement of such an effect with four superconducting gyroscopes carried onboard by a low-altitude polar spacecraft in the gravitational field of the Earth, but it is still unclear if it will be finally possible to meet the original accuracy because of some unexpected systematic aliasing effects occurred during the mission 3 . A test of gravito-magnetism 4 concerning the deflection of electromagnetic waves by Jupiter in its orbital motion has been performed in a dedicated radio-interferometric experiment (Fomalont and Kopeikin 2008). With regard to other suggested non-gravito-magnetic tests of general relativity in the jovian gravitational field, Hiscock and Lindblom (1979) proposed to measure the much larger gravito-electric Einstein pericenter precessions (Einstein 1915) of the natural satellites of Jupiter and Saturn. There exist also plans for performing a test of the light bending due to the Jupiter's monopole and quadrupole mass moments with the forthcoming astrometric mission GAIA (Crosta and Mignard 2006).

The Jupiter's proper angular momentum amounts to (Soffel et al. 2003)

$$
S \approx 6.9 \times 10^{38} \mathrm{~kg} \mathrm{~m}^{2} \mathrm{~s}^{-1}
$$

Table 1 and eq. (5) yield for Juno

$$
\begin{aligned}
& \dot{\Omega}_{\mathrm{LT}}=68.5{\operatorname{mas~} \mathrm{yr}^{-1}}^{-1} \\
& \dot{\omega}_{\mathrm{LT}}=0 .
\end{aligned}
$$

\footnotetext{
${ }^{3}$ See on the WEB http://einstein.stanford.edu/

${ }^{4}$ In this case, the mass currents inducing a gravito-magnetic action are not those related to the Jupiter's proper rotation (intrinsic gravito-magnetism), but are due to its translational orbital motion (extrinsic gravito-magnetism).
} 
which correspond to a shift $\Delta \nu$ of the cross-track component of the planetocentric position (Christodoulidis et al. 1988)

$$
\Delta \nu_{\mathrm{LT}}=a \sqrt{1+\frac{e^{2}}{2}} \sin i \Delta \Omega_{\mathrm{LT}}=572 \mathrm{~m}(T=1 \mathrm{yr})
$$

over the entire duration of the mission. A total accuracy of the order of 1-10 m with respect to the km-level of the past Jupiter missions in reconstructing the Juno's orbit in a planetocentric frame does not seem an unrealistic target (J.D. Anderson, private communication to the author, November 2008), although much work is clearly required in order to have a more firm answer. Note that a 1-10 m accuracy implies a $0.2-2 \%$ error in measuring the gravito-magnetic shift; this will be independently confirmed in the next Section. Thus, the possibility of detecting the Lense-Thirring effect with Juno's orbit seems worth of further consideration. Concerning the magnitude of the jovian gravito-magnetic field, it must be noted that in literature there are estimates for $S$ which point towards smaller values than eq. (5) by a factor 1.5 - 1.6; for example, Machida et al. (2008) yield

$$
S=4.14 \times 10^{38} \mathrm{~kg} \mathrm{~m}^{2} \mathrm{~s}^{-1}
$$

the ratio of eq. (5) to eq. (9), i.e. 1.6, is close to 1.5 coming from the ratio of $C / M R^{2}=2 / 5=0.4$, valid for a homogenous sphere, to $C / M R^{2}=0.264$ by Irwin (2003) who assumes a concentration of mass towards the Jupiter's center. Here we will consider

only the systematic uncertainty induced by the imperfect knowledge of the Newtonian part of the Jupiter's gravitational field; we will use eq. (5) for $S$.

\section{The systematic uncertainty due to the zonal harmonics of the Jupiter's gravitational potential}

A major source of systematic uncertainty is represented by the departures of the Jupiter's gravitational field from spherical symmetry. Indeed, the zonal $(m=0)$ harmonic 
coefficients $J_{\ell}$ of the multipolar expansion of the Newtonian part of the planet's gravitational potential give rise to long-period, that is averaged over one orbital revolution, orbital effects on the longitude of the ascending node $\Omega$, the argument of pericentre $\omega$ and the mean anomaly $\mathcal{M}$ (Kaula 1966)

$$
\begin{aligned}
\langle\dot{\Omega}\rangle & =\sum_{\ell=2} \dot{\Omega}_{\ell \ell} J_{\ell}, \\
\langle\dot{\omega}\rangle & =\sum_{\ell=2} \dot{\omega}_{\ell \ell} J_{\ell}, \\
\langle\dot{\mathcal{M}}\rangle & =\sum_{\ell=2} \dot{\mathcal{M}}_{\ell \ell} J_{\ell},
\end{aligned}
$$

where $\dot{\Omega}_{\ell \ell} \dot{\omega}_{\ell \ell}, \dot{\mathcal{M}}_{\ell}$ are coefficients depending on the planet's $G M$ and equatorial radius $R$, and on the spacecraft's inclination $i$ and eccentricity $e$ through the inclination $F_{\ell m p}(i)$ and eccentricity $G_{\ell p q}(e)$ functions, respectively (Kaula 1966). Note that one of the major scientific goals of the Juno mission is a greatly improved determination of just the harmonic coefficients of the jovian gravity potential; for the present-day values of the zonals for $\ell=2,3,4,6$ see Table 2. According to Anderson et al. (2004), it might be possible to

Table 2: Zonal harmonics of the Jupiter's gravity field according to the JUP230 orbit solution (Jacobson 2003) based on Galileo data.

\begin{tabular}{cccc}
\hline$J_{2}\left(\times 10^{6}\right)$ & $J_{3}\left(\times 10^{6}\right)$ & $J_{4}\left(\times 10^{6}\right)$ & $J_{6}\left(\times 10^{6}\right)$ \\
\hline $14696.43 \pm 0.21$ & $-0.64 \pm 0.90$ & $-587.14 \pm 1.68$ & $34.25 \pm 5.22$ \\
\hline
\end{tabular}

determine the first three even zonals with an accuracy of $10^{-9}$ and the other ones up to $\ell=30$ at a $10^{-8}$ level. Concerning $J_{2}$, this would be an improvement of two orders of magnitude with respect to Table 2, while the improvements in $J_{4}$ and $J_{6}$ would be of the 
order of three orders of magnitude. By using the results we are going to present below for the long-period node and pericenter precessions, it can be shown that determining the low degree zonals at $10^{-9}$ level of accuracy translates into an accuracy of the order of $0.5-1$ mas $\mathrm{yr}^{-1}$ in $\dot{\Omega}$ and $\dot{\omega}$, thus confirming the expectations of the previous Section.

For the long-period terms the condition

$$
\ell-2 p+q=0
$$

is fulfilled. Thus,

$$
\begin{aligned}
& \dot{\Omega}_{. \ell}=-\frac{n}{\sqrt{1-e^{2}} \sin i}\left(\frac{R}{a}\right)^{\ell} \sum_{p=0}^{\ell}\left[\frac{d F_{\ell 0 p}}{d i} G_{\ell p(2 p-\ell)} W_{\ell 0 p(2 p-\ell)}\right] \\
& \dot{\omega}_{. \ell}=-\frac{n}{\sqrt{1-e^{2}}}\left(\frac{R}{a}\right)^{\ell} \sum_{p=0}^{\ell}\left[-\cot i \frac{d F_{\ell 0 p}}{d i} G_{\ell p(2 p-\ell)}+\frac{\left(1-e^{2}\right)}{e} F_{\ell 0 p} \frac{d G_{\ell p(2 p-\ell)}}{d e}\right] W_{\ell 0 p(2 p-\ell)},(16) \\
& \dot{\mathcal{M}}_{. \ell}=n\left\{1-\left(\frac{R}{a}\right)^{\ell} \sum_{p=0}^{\ell} F_{\ell 0 p}\left[6 G_{\ell p(2 p-\ell)}-\frac{\left(1-e^{2}\right)}{e} \frac{d G_{\ell p(2 p-\ell)}}{d e}\right] W_{\ell 0 p(2 p-\ell)}\right\}
\end{aligned}
$$

where $W_{\ell 0 p(2 p-\ell)}$ are trigonometric functions having the pericentre as their argument and $n=\sqrt{G M / a^{3}}$ is the unperturbed Keplerian mean motion. Contrary to the case of small eccentricity satellites, in this case we will be forced to keep all the terms of order $\mathcal{O}\left(e^{k}\right)$ with $k>2$ in computing the eccentricity functions for given pairs of $\ell$ and $p$. Moreover, we will need also all the non-zero eccentricity and inclination functions for a given degree $\ell$, that is we will consider all the non-vanishing terms with $0 \leq p \leq \ell$. First, we will extend our calculations to the even zonals so far determined, i.e. $\ell=2,4,6$. In this case,

$$
W_{\ell 0 p(2 p-\ell)}=\cos [(\ell-2 p) \dot{\omega} t]=\cos (q \dot{\omega} t) .
$$

It must be noted that the terms with

$$
p=\frac{\ell}{2}, q=0
$$


yield secular precessions, i.e. $W_{\ell 0 \frac{\ell}{2} 0}=1$, while those with

$$
q=2 p-\ell \neq 0
$$

induces harmonic signals with circular frequencies $-q \dot{\omega}$.

For the degree $\ell=2$ the non-vanishing inclination and eccentricity functions and their derivatives are

$$
\begin{gathered}
F_{201}=\frac{3}{4} \sin ^{2} i-\frac{1}{2} . \\
\frac{d F_{201}}{d i}=\frac{3}{2} \sin i \cos i . \\
G_{210}=\frac{1}{\left(1-e^{2}\right)^{3 / 2}} . \\
\frac{d G_{210}}{d e}=\frac{2}{3} \frac{e}{\left(1-e^{2}\right)^{4 / 3}} .
\end{gathered}
$$

In this case, only secular precessions occur.

For $\ell=4$ we have

$$
\begin{gathered}
F_{401}=-\frac{35}{32} \sin ^{4} i+\frac{15}{16} \sin ^{2} i=F_{403}, \\
F_{402}=\frac{105}{64} \sin ^{4} i-\frac{15}{8} \sin ^{2} i+\frac{3}{8} . \\
\frac{d F_{401}}{d i}=\left(-\frac{35}{8} \sin ^{3} i+\frac{15}{8} \sin i\right) \cos i=\frac{d F_{403}}{d i}, \\
\frac{d F_{402}}{d i}=\left(\frac{105}{16} \sin ^{3} i-\frac{15}{4} \sin i\right) \cos i .
\end{gathered}
$$




$$
\begin{aligned}
G_{41-2} & =\frac{3}{4} \frac{e^{2}}{\left(1-e^{2}\right)^{7 / 2}}=G_{432} \\
G_{420} & =\frac{1+\frac{3}{2} e^{2}}{\left(1-e^{2}\right)^{7 / 2}} . \\
\frac{d G_{41-2}}{d e} & =\frac{3}{14} \frac{e\left(7-6 e^{2}\right)}{\left(1-e^{2}\right)^{8 / 7}}=\frac{d G_{432}}{d e} \\
\frac{d G_{420}}{d e} & =\frac{1}{7} \frac{e\left(23-18 e^{2}\right)}{\left(1-e^{2}\right)^{8 / 7}}
\end{aligned}
$$

In addition to secular terms, also harmonic signals with the frequencies $\pm 2 \dot{\omega}$ are present.

In the case of $\ell=6$ the inclination and eccentricity functions, along with their derivatives, are

$$
\begin{gathered}
F_{601}=\frac{693}{512} \sin ^{6} i-\frac{315}{256} \sin ^{4} i=F_{605}, \\
F_{602}=-\frac{3465}{1024} \sin ^{6} i+\frac{315}{64} \sin ^{4} i-\frac{105}{64} \sin ^{2} i=F_{604}, \\
F_{603}=\frac{1155}{256} \sin ^{6} i-\frac{945}{64} \sin ^{4} i+\frac{105}{32} \sin ^{2} i-\frac{5}{16} . \\
\frac{d F_{601}}{d i}=\left(\frac{2079}{256} \sin ^{5} i-\frac{315}{64} \sin ^{3} i\right) \cos i=\frac{d F_{605}}{d i} \\
\frac{d F_{602}}{d i}=\left(-\frac{10395}{512} \sin ^{5} i+\frac{315}{16} \sin ^{3} i-\frac{105}{32} \sin i\right) \cos i=\frac{d F_{604}}{d i}, \\
\frac{d F_{603}}{d i}=\left(\frac{3465}{128} \sin ^{5} i-\frac{945}{16} \sin ^{3} i+\frac{105}{16} \sin i\right) \cos i .
\end{gathered}
$$




$$
\begin{aligned}
G_{61-4} & =\frac{5}{16} \frac{e^{4}}{\left(1-e^{2}\right)^{11 / 2}}=G_{654} \\
G_{62-2} & =\frac{5}{2} \frac{e^{2}}{\left(1-e^{2}\right)^{11 / 2}}\left(1+\frac{e^{2}}{8}\right)=G_{642}, \\
G_{630} & =\frac{1}{\left(1-e^{2}\right)^{11 / 2}}\left(1+5 e^{2}+\frac{15}{8} e^{4}\right) . \\
\frac{d G_{61-4}}{d e} & =\frac{5}{4} \frac{e^{3}\left(1-\frac{21}{22} e^{2}\right)}{\left(1-e^{2}\right)^{12 / 11}}=\frac{d G_{654}}{d e} \\
\frac{d G_{62-2}}{d e} & =5 \frac{e\left(1-\frac{29}{44} e^{2}-\frac{21}{88} e^{4}\right)}{\left(1-e^{2}\right)^{12 / 11}}=\frac{d G_{642}}{d e} \\
\frac{d G_{630}}{d e} & =\frac{7}{44} \frac{e\left(64-10 e^{2}-45 e^{4}\right)}{\left(1-e^{2}\right)^{12 / 11}} .
\end{aligned}
$$

In addition to the secular rates, also harmonic signals with frequencies $\pm 4 \dot{\omega}, \pm 2 \dot{\omega}$ are present.

Let us, now, focus on the action of the odd $(\ell=3,5,7, \ldots)$ zonal $(m=0)$ harmonics. In this case,

$$
W_{\ell 0 p(2 p-\ell)}=\sin [(\ell-2 p) \dot{\omega} t]=-\sin (q \dot{\omega} t),
$$

so that only harmonic terms occur for $q \neq 0$.

For $\ell=3$ we have 


$$
\begin{gathered}
F_{301}=\frac{15}{16} \sin ^{3} i-\frac{3}{4} \sin i=-F_{302} \\
\frac{d F_{301}}{d i}=\left(\frac{45}{16} \sin ^{2} i-\frac{3}{4}\right) \cos i=-\frac{d F_{302}}{d i} . \\
G_{31-1}=\frac{e}{\left(1-e^{2}\right)^{5 / 2}}=G_{321} \\
\frac{d G_{31-1}}{d e}=\frac{1-\frac{3}{5} e^{2}}{\left(1-e^{2}\right)^{6 / 5}}=\frac{d G_{321}}{d e} .
\end{gathered}
$$

Thus, we have long-period effects varying with the frequencies $\pm \dot{\omega}$.

Concerning the even and odd zonal long-period harmonic terms, it must be noted that they can be approximated by secular precessions with a high level of accuracy over the expected 1-yr lifetime $T$ of Juno because the period of its periJove is of the order of $\approx 500$ yr, i.e.

$$
\begin{gathered}
\cos (q \omega)=\cos \left(q \dot{\omega} t+q \omega_{0}\right) \approx \cos \left(q \omega_{0}\right), 0 \leq t \leq T, \\
\sin \omega=\sin \left(\dot{\omega} t+\omega_{0}\right) \approx \sin \omega_{0}, 0 \leq t \leq T .
\end{gathered}
$$

Thus, the choice of the initial condition $\omega_{0}$ will be crucial in determining their impact.

Now, it would be possible, in principle, to use the node of Juno to measure the gravito-magnetic effect. Indeed, the Lense-Thirring node precession is independent of $i$, while all the zonal precessions of $\Omega$ vanish for $i=90$ deg. It does not occur for the periJove and the mean anomaly, but they are not affected by the gravito-magnetic force for $i=90 \mathrm{deg}$. In reality, the situation will be likely different because of the unavoidable 
orbit injection errors which will induce some departures $\delta i$ of the Juno's orbital plane from the ideal polar configuration. Thus, unwanted, corrupting node zonal secular precessions will appear; their mis-modeling due to the uncertainties $\delta J_{\ell}$ may swamp the recovery of the Lense-Thirring effect if their determination by Juno will not be accurate enough. Note that there is no risk of some sort of a-priori 'imprint' effect of the Lense-Thirring effect itself on the values of the zonals retrieved from the Juno's periJove motion because the gravito-magnetic pericenter precession vanishes for polar orbits.

By assuming the values quoted in Table 2 for the uncertainties $\delta J_{\ell}, \ell=2,3,4,6$, let us see what is the impact of a non-perfectly polar orbital geometry on the node Lense-Thirring precessions. The results are depicted in Figure 1 for each degree $\ell$ separately; the initial condition $\omega_{0}=90 \mathrm{deg}$ has been used. It should be noted that, in view of the likely correlations among the determined zonals, a realistic upper bound of the total bias due to them can be computed by taking the linear sum of each mis-modeled terms. The major source of bias is the so far poorly known $J_{6}$; an improvement of four orders of magnitude, which sounds rather unlikely to be obtained even with Juno (Anderson et al. 2004), would be required to push its aliasing effect at a percent level of the Lense-Thirring effect. The situation for the other zonals is more favorable; $J_{4}$ should be known better than now by a factor 1000, which is, instead, a realistic goal according to Anderson et al. (2004). Thus, we conclude that a nearly-polar orbit $1 \mathrm{deg}$ off the ideal $90 \mathrm{deg}$ case would likely prevent to obtain a measurement of the gravito-magnetic node precession at a decent level of accuracy.

Thanks to the high eccentricity of the Juno's orbit, also the periJove and the mean anomaly are well defined, so that they can be used in a suitable way to remove the bias of 


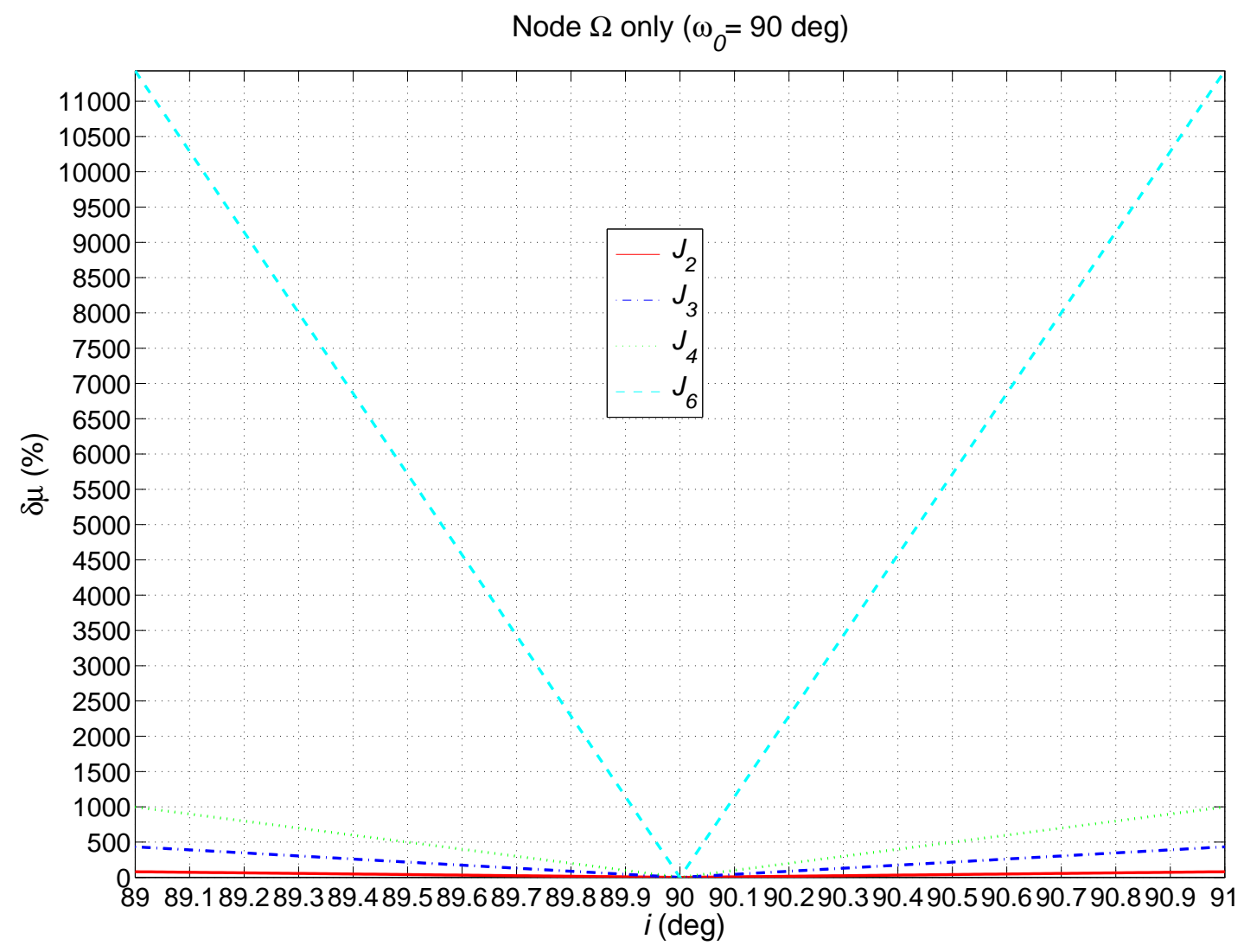

Fig. 1. - Systematic percent bias on the Lense-Thirring node precession induced by the mis-modeling in the zonals $J_{2}, J_{3}, J_{4}, J_{6}$ according to Table 2 for $89 \mathrm{deg} \leq i \leq 91 \mathrm{deg}$ and $\omega_{0}=90 \mathrm{deg}$. 
$J_{6}$ and $J_{2}$. Let us write down

$$
\begin{aligned}
\delta \dot{\Omega} & =\dot{\Omega}_{.2} \delta J_{2}+\dot{\Omega}_{.6} \delta J_{6}+\mu \dot{\Omega}_{\mathrm{LT}}+\Delta_{\Omega}, \\
\delta \dot{\omega} & =\dot{\omega}_{.2} \delta J_{2}+\dot{\omega}_{.6} \delta J_{6}+\mu \dot{\omega}_{\mathrm{LT}}+\Delta_{\omega}, \\
\delta \dot{\mathcal{M}} & =\dot{\mathcal{M}}_{.2} \delta J_{2}+\dot{\mathcal{M}}_{.6} \delta J_{6}+\Delta_{\mathcal{M}} .
\end{aligned}
$$

Here $\delta \dot{\Psi}$ denotes some sort of Observed-minus-Calculated $(O-C)$ quantity for the rate of the Keplerian element $\Psi$ which accounts for every unmodeled/mis-modeled dynamical effects; it may be, for example, a correction to the modeled precessions to be phenomenologically estimated as a solve-for parameter of a global fit of the Juno's data, or it could be a computed time-series of "residuals" of $\Psi$ by suitably overlapping orbital arcs. The gravito-magnetic force should be purposely not modeled in order to be fully present in $\delta \dot{\Psi}$. The parameter $\mu$ i i 61 in GTR and 0 in Newtonian mechanics and accounts for the Lense-Thirring effect. The $\Delta$ terms include all the other systematic errors like the precessions induced by the mis-modeled parts of the second even zonal harmonic $\delta J_{4}$ and the first odd zonal harmonic $\delta J_{3}$, the mis-modeling due to the uncertainty in Jupiter's $G M$, etc. By solving for $\mu$ one obtains

$$
\delta \dot{\Omega}+c_{1} \delta \dot{\omega}+c_{2} \delta \dot{\mathcal{M}}=\dot{\Omega}_{\mathrm{LT}}+c_{1} \dot{\omega}_{\mathrm{LT}}+\Delta,
$$

\footnotetext{
${ }^{5}$ Since the Keplerian elements are not directly measurable quantities, we use here the term "residual" in an improper sense.

${ }^{6}$ It is not one of the standard PPN parameters, but it can be expressed in terms of $\gamma$ as $\mu=(1+\gamma) / 2$.
} 
with

$$
\begin{aligned}
& c_{1}=\frac{\dot{\mathcal{M}}_{.6} \dot{\Omega}_{.2}-\dot{\Omega}_{.6} \dot{\mathcal{M}}_{.2}}{\dot{\omega}_{.6} \dot{\mathcal{M}}_{.2}-\dot{\mathcal{M}}_{.6} \dot{\omega}_{.2}}, \\
& c_{2}=\frac{\dot{\Omega}_{.6} \dot{\omega}_{.2}-\dot{\omega}_{.6} \dot{\Omega}_{.2}}{\dot{\omega}_{.6} \dot{\mathcal{M}}_{.2}-\dot{\mathcal{M}}_{.6} \dot{\omega}_{.2}} .
\end{aligned}
$$

The combination of eq. (55), with eq. (56) and eq. (57), is designed, by construction, to single out the combined Lense-Thirring precessions and to cancel out the combined seculan 7 precessions due to $J_{2}$ and $J_{6}$ along with their mis-modeling. Instead, it is affected by $\Delta$ which acts as a systematic bias on the Lense-Thirring signal of interest. $\Delta$ globally includes the mis-modeled part of the combined precessions induced by $J_{3}$ and $J_{4}$; the sources of uncertainty reside in $J_{3}$ and $J_{4}$ themselves and in the Jupiter's $G M$ through the mean motion $n$ which enters the coefficients $\dot{\Omega}_{\ell \ell}, \dot{\omega}_{. \ell}, \dot{\mathcal{M}}_{. \ell}$.

In Figure 2 the impact of the mis-modeling in $J_{3}$ and $J_{4}$ for $\omega_{0}=90$ deg is depicted. In Figure 3 we use $\omega_{0}=0$ deg. In this case the situation is much more favorable because for a total departure of $\pm 1 \mathrm{deg}$ from $i=90 \mathrm{deg}$, an improvement of only two orders of magnitude in $J_{3}$, which is, today, still compatible with zero, and $J_{4}$ would be needed to reach the percent level; let us recall that the expected improvement in $J_{4}$ with respect to the results by Jacobson (2003) is of three orders of magnitude (Anderson et al. 2004). Note that a value of $\omega_{0}$ far from $90 \mathrm{deg}$ is preferable to minimize the perturbations.

Another potential source of systematic error is the Jupiter's GM whose uncertainty $\delta(G M)$ indirectly affects eq. (55) through the Keplerian mean motion $n$ entering the uncanceled $J_{3}$ and $J_{4}$ combined precessions; $\delta n$ is also present via the mean anomaly itself. However, it turns out that it is of no concern because, according to the present-day level of

\footnotetext{
${ }^{7}$ We include in them also the long-period harmonic terms for the reasons explained before.
} 


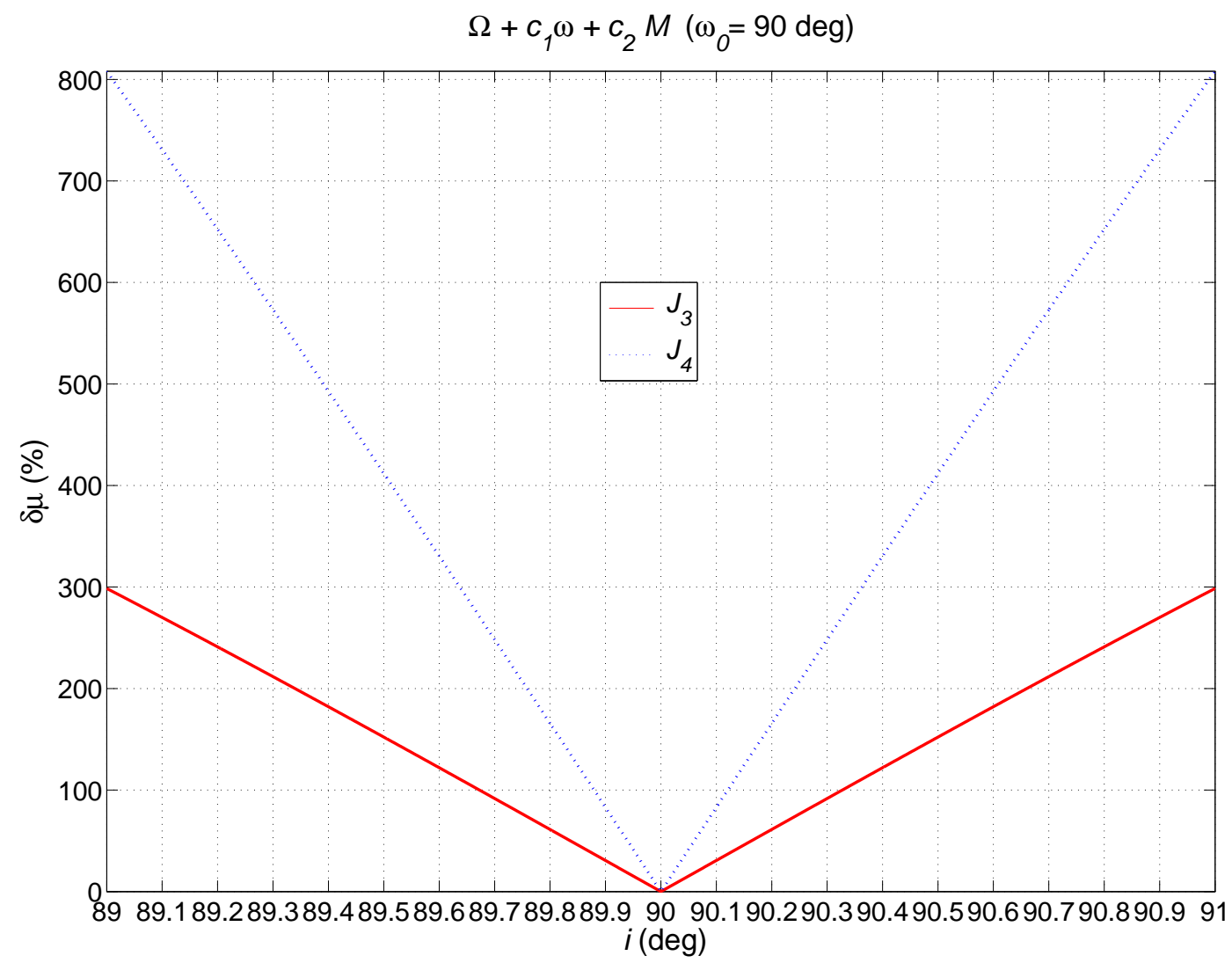

Fig. 2.- Systematic percent bias on the Lense-Thirring precessions, combined according to eq. (55), induced by the mis-modeling in the uncanceled zonals $J_{3}, J_{4}$ (Table 2) for $89 \mathrm{deg} \leq i \leq 91 \mathrm{deg}$ and $\omega_{0}=90$ deg. 


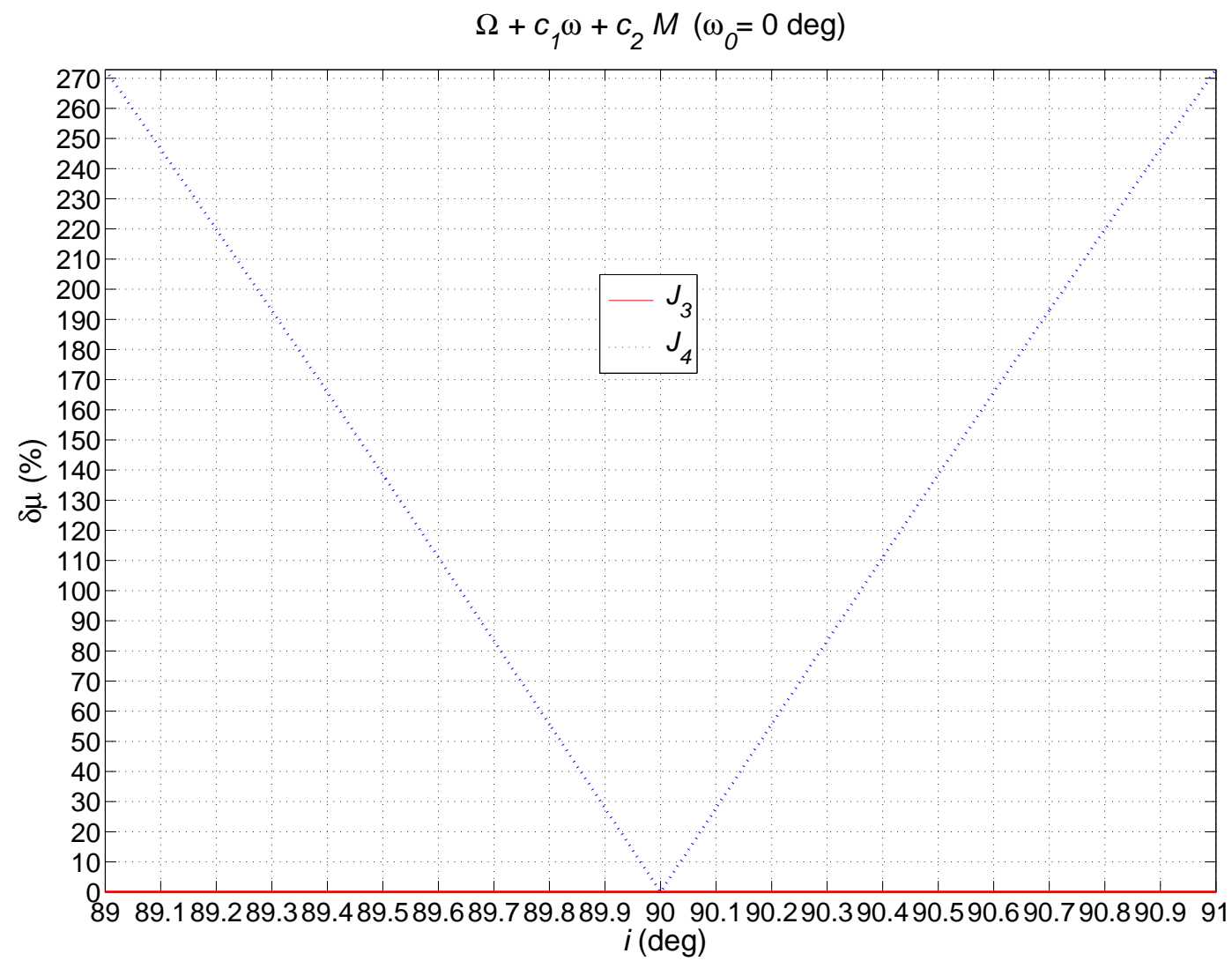

Fig. 3. - Systematic percent bias on the Lense-Thirring precessions, combined according to eq. (55), induced by the mis-modeling in the uncanceled zonals $J_{3}, J_{4}$ (Table 2) for $89 \mathrm{deg} \leq i \leq 91 \mathrm{deg}$ and $\omega_{0}=0$ deg. 
relative uncertainty (Jacobson 2003)

$$
\frac{\delta(G M)}{G M}=1.6 \times 10^{-8}
$$

its impact on the combined Lense-Thirring precessions is well below the percent level.

\section{Discussion and conclusions}

The node $\Omega$ of Juno, a recently approved spacecraft aimed to orbit Jupiter along a highly eccentric $\left(r_{\min }=1.06 R_{\mathrm{Jup}}, r_{\max }=39 R_{\mathrm{Jup}}\right)$, polar $(i=90 \mathrm{deg})$ trajectory during one year to accurately map, among other things, its gravitational field, will be displaced by the general relativistic gravito-magnetic Lense-Thirring effect by about $572 \mathrm{~m}$ over the entire duration of the mission. A meter-level accuracy in determining the jovicentric orbit of Juno should not be an unrealistic goal to be reached. Equivalently, the gravito-magnetic

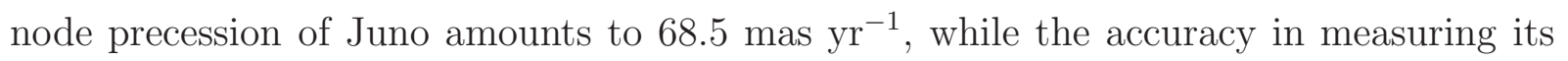

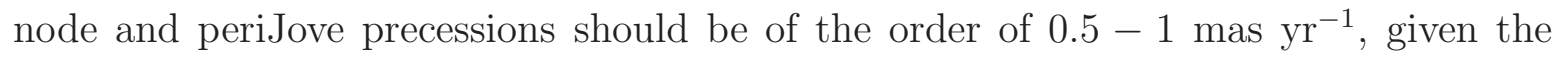
expected improvements in our knowledge of the departure of the jovian gravitational field from spherical symmetry. If the Juno's orbit was perfectly polar, the long-period node precessions induced by the zonal harmonics $J_{\ell}, \ell=2,3,4,6, \ldots$ of the non-spherical jovian gravitational potential would vanish, thus removing a major source of systematic alias on the Lense-Thirring secular precession. In reality, unavoidable orbit injection errors will displace the orbital plane of Juno from the ideal polar geometry; as a consequence, the mis-modeled part of the node zonal precessions would overwhelm the relativistic signal for just $\delta i= \pm 1 \mathrm{deg}$, in spite of the expected improvements in $J_{\ell}, \ell=2,4,6$ of three orders of magnitude. A suitable linear combination of the node, the periJove $\omega$ and the mean anomaly $\mathcal{M}$ will allow to cancel out the effect of $J_{2}$ and $J_{6}$; the remaining uncanceled $J_{3}$ and $J_{4}$ will have an impact on the Lense-Thirring combined precessions which should be reduced down to the percent level or better by the improved low-degree zonals. If one 
assumes the existence of gravito-magnetism as predicted by general relativity, the proposed measurement can also be considered as a direct, dynamical determination of the jovian proper angular momentum $S$ by means of the Lense-Thirring effect.

\section{Acknowledgments}

I thank J.D. Anderson for useful discussions. 


\section{REFERENCES}

Anderson, J.D., Lau, E.L., Schubert, G., Palguta, J.L., 2004. Gravity Inversion Considerations for Radio Doppler Data from the JUNO Jupiter Polar Orbiter. American Astronomical Society, DPS meeting \#36, \#14.09 http://aas.org/archives/BAAS/v36n4/dps2004/158.htm

Christodoulidis, D.C., Smith, D.E., Williamson, R.G., Klosko, S.M., 1988. J. Geophys. Res. 93(B6), 6216

Ciufolini, I. and Pavlis, E.C., 2004. Nature 431958

Crosta, M.T., and Mignard, F., 2006. Class. Quantum Grav. 234853

Einstein, A., 1915. Sitzber. Kön. Preuss. Akad. Berlin 47831

Everitt, C. W. F., 1974. The Gyroscope Experiment I. General Description and Analysis of Gyroscope Performance in Proc. Int. School Phys. "Enrico Fermi" Course LVI Bertotti, B. (ed.) (New York: New Academic Press) pp. 331-360.

Fomalont, E.B., and Kopeikin, S.M., 2008. Radio interferometric tests of general relativity in A Giant Step: from Milli- to Micro-arcsecond Astrometry Proceedings IAU Symposium No. 248, 2007 Jin, W.J., Platais, I., and Perryman, M.A.C. (eds.) (Cambridge University Press, 2008), pp. 383-386.

Guillot, T., 2005. Ann. Rev. Earth Planet. Sci. 33493

Haas, M. R., and Ross, D. K., 1975. Astrophys. Space Sci. 323

Hiscock, W.A., and Lindblom, L., 1979. Astroph. J. 231224 
Hori, Y., Sano, T., Ikoma, M., Ida, S., 2008. in: Sun, Y.-S., Ferraz-Mello, S., Zhou, J.-L. (eds.), EXOPLANETS: Detection, Formation and Dynamics Proceedings IAU Symposium No. 249, pp. 119-122.

Iorio, L., 2006. Class. Quantum Gravit. 23, 5451

Iorio, L. (ed.), 2007. The Measurement of Gravitomagnetism: A Challenging Enterprise. (NOVA, Hauppauge)

Iorio, L., 2008. Scholarly Research Exchange 2008, 105235

Iorio, L., 2009a. Space Science Reviews, doi:10.1007/s11214-008-9478-1, arXiv:0809.1373v2 $[\mathrm{gr}-\mathrm{qc}]$

Iorio, L., 2009b. Adv. Space Res., doi:10.1016/j.asr.2008.10.016, arXiv:0802.2031v7 [gr-qc]

Iorio, L., and Lainey, V., 2005. Int. J. Mod. Phys. D 142039

Irwin, P., 2003. Giant Planets of Our Solar System (Springer)

Jacobson, R.A., 2003. JUP230 orbit solution

Kaula, W.M., 1966. Theory of Satellite Geodesy (Blaisdell, Waltham)

Krogh, K., 2007. Class. Quantum Grav. 24, 5709

Lense, J. and Thirring, H., 1918. Phys. Z. 19156 Translated and discussed in Mashhoon, B., Hehl, F.W., Theiss, D.S., 1984. Gen. Relativ. Gravit. 16 711. Reprinted in Ruffini, R.J., Sigismondi, C. (eds), 2003. Nonlinear Gravitodynamics (Singapore: World Scientific) pp 349-88,

Lichtenegger, H.I.M. and Iorio, L., 2007. Post-Newtonian Orbital Perturbations in Iorio L (ed.) The Measurement of Gravitomagnetism: A Challenging Enterprise (Hauppauge, New York: NOVA publishers) pp. 87-100. 
Machida, M.N., Kokubo, E., Inutsuka, S., Matsumoto, T., 2008. Astroph. J. 6851220

Mashhoon, B., 2007. Gravitoelectromagnetism: A Brief Review in Iorio, L. (ed.) The Measurement of Gravitomagnetism: A Challenging Enterprise (Hauppauge, New York: NOVA publishers) pp. 29-39.

Matousek, S, 2007. Acta Astronautica 61932

Pugh, G.E., 1959. WSEG Research Memorandum No. 11

Ries, J.C., Eanes, R.J., Watkins, M.M., 2008. Confirming the Frame-Dragging Effect with Satellite Laser Ranging 16th Int. Laser Ranging Workshop, (Poznań (PL) 13-17 October 2008).

Schiff, L., 1960. Phys. Rev. Lett. 4215

Soffel, M., Klioner, S.A., Petit, G., et al., 2003. Astron. J. 126, 2687 